\title{
Multislice Computed Tomographic Findings of a Pseudoaneurysm of a Saphenous Vein Graft
}

\author{
Shigeru Naono, Akira Tamura and Junichi Kadota
}

Key words: multislice computed tomography, saphenous vein graft, pseudoaneurysm

(DOI: 10.2169/internalmedicine.46.6124)

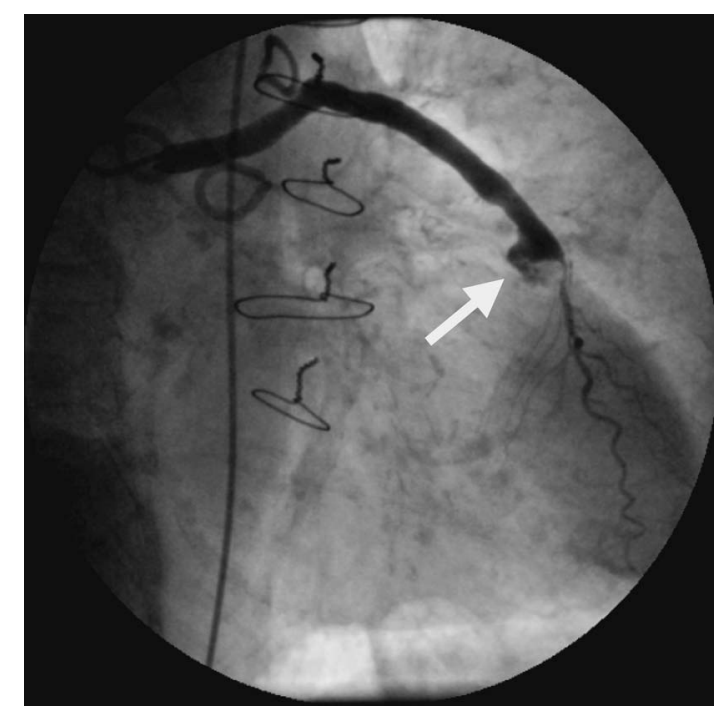

Picture A. Coronary angiogram showing an aneurysm of the saphenous vein graft to the left anterior descending coronary artery (arrow) and a severe stenosis at the site of the distal anastomosis.
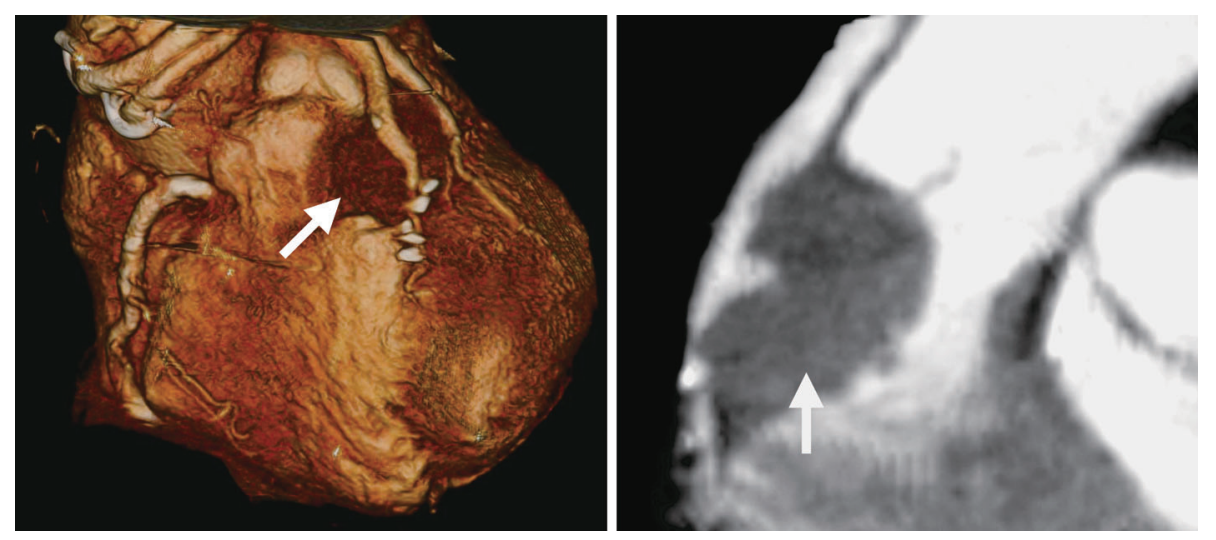

Picture B. Multislice computed tomography (left panel $=$ volume rendering image, right panel = maximum intensity projecting image) showing a pseudoaneurysm $(23 \times 26 \times 26 \mathrm{~mm})$ of the saphenous vein graft to the left anterior descending coronary artery (arrow). Note that only the neck of the pseudoaneurysm is dyed. 
An 83-year-old-woman was admitted because of recurrent anginal attacks. She had undergone coronary artery bypass surgery 10 years earlier. Coronary angiography revealed an aneurysm of the distal portion of the saphenous vein graft $(\mathrm{SVG})$ to the left anterior coronary artery (LAD) and a severe stenosis at the distal anastomostic site (Picture A). Multislice computed tomography showed a mass beneath the distal portion of the SVG to the LAD. The mass was hardly dyed by contrast medium (Picture B). These findings indicate that it is a pseudoaneurym of the SVG and that its cavity is almost obstructed by thrombus. To the best of our knowledge, this is the first case report describing multislice computed tomographic findings of a pseudoaneurym of an SVG.

\section{(C) 2007 The Japanese Society of Internal Medicine http://www.naika.or.jp/imindex.html}

